\title{
Benign tumour of the ampulla of Vater presenting with portal hypertension
}

\author{
Peter Aitken, Lindsay Erwin and James D. Fulton \\ Department of Geriatric Medicine, Stobhill General Hospital, Glasgow G21 3UW, UK.
}

\begin{abstract}
Summary: Benign tumours of the ampulla of Vater are uncommon and usually present with obstructive jaundice. This is believed to be the first report of anicteric portal hypertension and hypersplenism caused by a benign ampullary tumour.
\end{abstract}

\section{Introduction}

The prevalence of benign tumours of the ampulla of Vater is unknown as many are asymptomatic. ${ }^{1}$ Symptomatic presentation is usually with obstructive jaundice, which is frequently intermittent, and which may be associated with cholangitis. We present an 83 year old woman with anicteric portal hypertension and hypersplenism caused by a benign tumour of the ampulla of Vater.

\section{Case report}

An 83 year old woman was admitted in May 1987 with a history of recurrent falls. Her past medical history included a cholecystectomy in 1965 and a 40 year history of generalized seizures. She had remained seizure-free for over 3 years on sodium valproate $200 \mathrm{mg} /$ day. Physical examination was unremarkable.

Pancytopenia was noted (white cell count $3.1 \times 10^{9} / 1$; haemoglobin $9.3 \mathrm{~g} / \mathrm{dl}$, normochromic and normocytic; platelets $\left.95 \times 10^{9} / 1\right)$. Her liver function tests were deranged, the alkaline phosphatase and gamma glutamyl transpeptidase being considerably elevated and the transaminases slightly so; the serum bilirubin was normal. Autoantibody and viral screening was negative and assessment of her serum ferritin, vitamin $B_{12}$ and red cell folate was normal. An isotope liver scan demonstrated areas of reduced uptake in her liver associated with a moderately enlarged spleen. Ultrasound confirmed the presence of splenomegaly and revealed substantial dilatation of the intrahepatic ducts, the common bile duct $(1.9 \mathrm{~cm}$ diameter $)$ and the pancreatic duct. The pancreas appeared otherwise

Correspondence: James D. Fulton, M.B., Ch.B., M.R.C.P. (UK)

Accepted: 26 May 1988 normal. Her physical rehabilitation was successful and it was decided not to investigate her further at that stage unless her liver function deteriorated. She was discharged in July but was readmitted in September with a urinary tract infection and a further derangement of her liver function tests. Endoscopic retrograde cholangio-pancreatography (ERCP) was carried out and demonstrated oesophageal varices and what appeared to be an ampullary carcinoma. It was not possible to cannulate the ampulla of Vater. Biopsies were taken but, despite sectioning at several levels, no evidence of carcinoma was discovered, the appearances being those of a benign tubulovillous epithelial tumour. Moderate cellular atypia was present but no invasion was noted. ERCP was not repeated. Surgical exploration and attempted resection was felt not to be justified in the presence of thrombocytopenia, oesophageal varices and possible secondary biliary cirrhosis. For the same reasons liver biopsy was not attempted and she remains asymptomatic whilst being managed conservatively with fat-soluble vitamin supplements.

\section{Discussion}

Benign tumours of the ampulla of Vater are uncommon but are all considered to have malignant potential. ${ }^{2}$ The growth of these tumours is generally slow and they rarely exceed $2 \mathrm{~cm}$ in diameter. The history is often chronic and may extend over 20 years. This patient's biliary tract is presumed to have been normal at the time of her cholecystectomy in 1965. However, in the light of the chronicity of this condition, it is conceivable that the tumour may have been of aetiological significance in the development of her cholecystitis.

(C) The Fellowship of Postgraduate Medicine, 1988 
The mechanism of development of her portal hypertension is open to speculation. Portal hypertension has previously been reported in association with cholangiocarcinoma in 3 patients by Gold and colleagues. $^{3}$ One of their patients had been receiving thioridazine and imipramine, both of which have recorded hepatotoxicity. Our patient had been receiving sodium valproate at low dose. Idiosyncratic hepatic damage due to this drug is rare and usually occurs within the first 6 months. It is considered unlikely that the portal hypertension in this patient was due to valproate hepatotoxicity, and that the occlusive biliary tumour was incidental, as the bile ducts were dilated. In Gold's patients, the presentation of portal venous obstruction was more classical than the presentation of the above patient. In particular, all 3 patients developed significant jaundice. A possible explanation for the essentially anicteric presentation of our patient may be the

\section{References}

1. Bouchier, I.A.D., Allan, R.W., Hodgson, H.J.F. \& Keighley, M.R.B. Textbook of Gastroenterology. Bailliere Tindall, Edinburgh, London, 1984, 1461-1472.

2. Cattell, R.B., Braasch, J.W. \& Kahn, F. Polypoid epithelial tumours of the bile ducts. $N$ Engl Med J 1962, 266: 57-61. presumed chronicity of her illness. This favours an alternative aetiology - gradually progressive $\stackrel{\mathbb{Q}}{\Omega}$ secondary biliary cirrhosis due to biliary obstruc- $C$. tion by the benign tumour. The gradual onset may $\overrightarrow{\vec{F}}$ similarly reduce the risk of other acute complications of biliary disease such as pancreatitis, which is well documented as a complication of ampullary tumours. ${ }^{4}$ Finally, it is also unlikely that any acute pathology, for example, portal venous thrombosis, could be held accountable for her condition.

In summary, we present the first recorded case of anicteric portal hypertension and hypersplenism due to biliary obstruction by a benign tumour occurring in an elderly woman.

\section{Acknowledgements}

We wish to thank Dr J. Forrest for performing the ERCP and Dr G.D. Smith for his histological expertise.

3. Gold, J.A., Sostman, H.D. \& Burrell, M.I. Cholangiocarcinoma with portal venous obstruction. Diagn $\mathbb{(}$ Radiol 1979, 130: 15-20.

4. Dixon, J.M., Chapman, R.W. \& Berry, A.R. Carcinoid tumour of the ampulla of Vater presenting as acute pancreatitis. Gut 1987, 28: 1296-1297. 